\title{
Production Optimization versus Asset Availability - a Review
}

\author{
${ }^{1,2}$ Balduíno Mateus; ${ }^{3,4}$ José Torres Farinha; ${ }^{2}$ António Marques Cardoso \\ ${ }^{1}$ Industrial Eng. and Management, Univ. Lusofona. Lisbon, Portugal \\ ${ }^{2}$ CISE - Electromechatronic Systems Research Centre, Univ. Beira Interior, Portugal \\ ${ }^{3}$ CEMMPRE - Centre for Mechanical Engineering, Materials and Processes, Univ. of Coimbra, Portugal \\ ${ }^{4}$ ISEC/IPC - Polytechnic Institute of Coimbra, Portugal
}

\begin{abstract}
Nowadays, companies want to give a quick answer in order to face their market competitors. These quick responses must be reflected in the quality of the products; to this be possible, it is necessary to manage a number of factors that will bring benefits in its market positioning. As technology grows, there is the possibility, at a computational level, to create a combination of mathematical and technological tools that were not implemented in the past due to the lack of resources, since they have high robustness about their analytical resolution.

This paper presents mathematical and computer tools that have potential great benefits when applied to industrial problems solving, such as operation management.

Along the paper it is made a temporal location of all tools with their main objectives about optimizing industrial processes, focusing on maintenance costs, contributing directly to the rationalization of global costs of the processes.

Analytical and technological methods that have had great success regarding to the reduction costs of production in industries are presented. The approaches of this paper bring a literary review of process optimization, namely about Neural Networks and multivariate analysis for prediction.
\end{abstract}

Key-Words: Optimization; Production; Forecast; Neural Networks; Multivariate Analysis; Industrial Maintenance

Received: March 8, 2020. Revised: July 10, 2020. Accepted: July 31, 2020. Published: August 18, 2020.

\section{Introduction}

The research on maintenance optimization has been a priority [1], also having been a big trend in the area of optimization based on maintenance simulation [2], [3]. The research in this context has as its main objective to find the best maintenance plans that minimize the general maintenance cost or maximize a system performance measure bringing cost reductions [4]. Maintenance plays an important role in the industry, being responsible for improving the availability of assets, thus reducing the downtime in industrial plants. The cost of maintenance can vary between the values of $15 \%$ and $70 \%$ of the production costs [5]. With the technological evolution of industrial processes, maintenance has increased its complexity [5], [6]. This is mostly due to modern manufacturing systems that involve numerous interactions and dependencies among components [7]. Corrective and preventive maintenance aim to take the systems "as good as new". Regarding the predictive substitution model policy, under some assumptions, the one that fits most practical situations, has proved to have a limit control policy [8].

J. Khalil [9] Predicting the failure rate of each machine part is possible. Because of this, the following actions must be executable, spending the minimum effort:

- get a clear view of the industrial domain (usually vast, confusing and complicated) in an indus- try, in terms of its most basic units (the machine parts);

- obtain an economical and scientifically calculated service life for each machine part in the industrial domain;

- to adapt preventive actions as opportunities;

- to be able to refine restricted production, subject to the relationship between recent and historical trends in machine part failures; and

- to obtain a mathematical formulation of the costs and the probabilities of survival of each part of the machine throughout its useful life, in order to be able to change the availability of that part with full knowledge of the financial consequences.

The structure of maintenance cycles in the preventive strategy makes the objective function of the decisionmaking problem discontinuous. Therefore, it is suggested to solve the problem with the use of dynamic programming methods.

In the development of a dedicated computer program, it was possible to highlight possible resolutions for decision-making problems in relation to: manner, scope and schedules of replacements, repairs and regular maintenance of elements of technical objects, mode and schedules of diagnosis and preventive replacement of elements and problems of supplying spare parts to the maintenance subsystem [10]. 
Based on the inspiration of [11], three slightly different decision dimensions are proposed: "product dimension"; "risk dimension"; and "resource dimension". This research concludes that these decision dimensions must be considered simultaneously, at the same time, in which they optimize maintenance in an integrated way. The structure of this paper is the following:

- The first section presents the introduction of the theme;

- The second section presents the state of the art, namely a global approach about optimization;

- The third section presents proposals for possible resolution of the problems in this area;

- The fourth section presents a discussion based on the results of the research;

- Finally, they are presented the conclusions.

The degree of adaptation of a competitive organization imply priorities in its primary decisions related to structural and infrastructural investments, providing the key to the progress of the full potential of their operations as a competitive weapon. Figure 1 illustrates a graphic model about the operations strategy generally accepted [12].

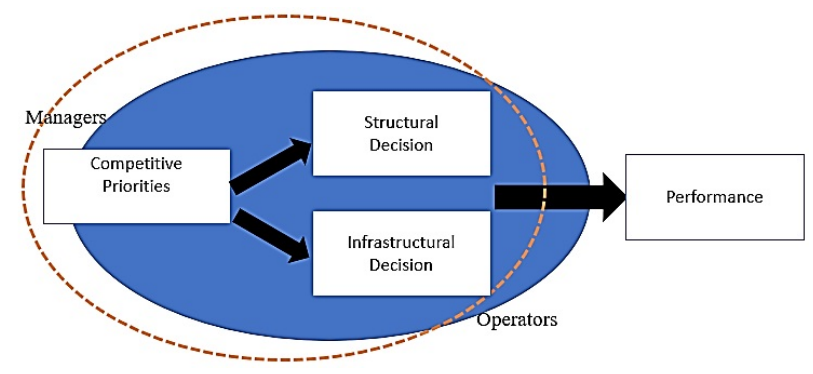

Figure 1: Operations strategy model [12]

The paper is focused on the aggregation of knowledge for future implementations of the tools covered in the scope of operations management; in the second section it is made a retrospective of the premises of the maintenance policies focused on production costs that supports the paper case studies. In this same section, the forecasting tools, based on multivariate analysis and neural networks are addressed. In section three the proposal for the next phase of the study is presented and, in third section, the state of the art of these tools is presented; in section five a conclusion of the study is made.

\section{State of the Art}

\subsection{Global approach}

The poor maintenance of our car can lead its poor performance, causing malfunction at an inopportune moment, even causing a bad flow on the road. An operation's manager does not want this happen on his manufacturing line. Therefore, it can be said that maintenance is a fundamental pillar for the fluidity of the production plant. This paper focuses on maintenance management and in what it can prevent failure and increase equipment availability. Compared to the costs associated with maintenance, the production system, according to Dhillon [13] becomes increasingly important, because, in many industrial plants, the maintenance costs can exceed $30 \%$ of the operational costs and, in the context of the service life cycle, maintenance and support of manufacturing systems, represent 60 to $75 \%$ of the total cost of the life cycle. According to Mobley [14], the production capacity of a plant is partly demarcated by the availability of production systems and their auxiliary equipment, being the main function of the maintenance organization to ensure that all equipment and systems in the plant are up to date, always "online" and in good operating conditions, without jeopardizing profitability and, always possible using other tools such as optimization. Although it seems that optimization is an activity present in people's daily lives, according to Cua, McKone \& Schroeder [15], this is the best option to carry out a task without compromising restrictions: a fast and efficient task that companies use to improve and make more profitable processes. The same authors say that, the optimization techniques seek the best solution for each problem (maximum or minimum of measurable quantities in their domains of definition); they are necessary tools in many areas of engineering, such as:

- Operational research - optimization of technical and economic systems, stock control, production planning, etc.;

- Process engineering - process sizing and optimization, parameter estimation, data grouping, flexibility analysis, etc.;

- Process control - system identification, optimal control, adaptive control, predictive control, state estimators, etc.;

- Numerical analysis - approximations, regression, solution of linear and non-linear systems, etc.

Wang et al. [16] say that an optimization process is needed to determine the optimal capabilities and operational strategy. There has been a great contribution in making optimization in a dynamic way by combining process simulators with metaheuristic techniques 
for simultaneous optimization of process flowcharts with the corresponding operating conditions [17]. The Operations Management have applications into strategic and tactically oriented applications, namely in next areas: aggregate planning; forecasting; location decisions; scheduling; capacity planning; layout; process and product design; quality control; task design; control inventory; maintenance and reliability [18].

In the industrial area, it is also possible to carry out an adaptation of the optimization model, demonstrating their efficiency for the solution of complex problems; it can start with simpler problems, observing what already exists in nature, for example, [19] retransmitting a study that presents an overview of recent work on ant algorithms, i.e., algorithms for discrete optimization that were inspired by the observation of ant colony forage behaviour and presenting the ant colony optimization metaheuristic.

The field of metaheuristics for applying combinatorial optimization problems is a rapidly growing field of research. This is due to the importance of combinatorial optimization problems for the scientific and industrial world [20]. Alaswad \& Xiang [21] present a review of the Condition Based Maintenance (CBM) literature with emphasis on mathematical modelling and optimization approaches. They focused the review on important aspects of the CBM, such as optimization criteria, frequency of inspection, degree of maintenance, solution methodology, etc.

Nocedal \& Wright [22] refer that knowledge of the capabilities and limitations of optimization algorithms leads to a better understanding of their impact in various applications and points the way for future research on algorithms and software for improvement and extension. To encompass the optimization of a digital industry, it is necessary to fulfil some requirements such as the robustness of the database and the reliability of the data/samples. According to Dekker [1], there are several applications of maintenance optimization models that generally cover four aspects:

1. A description of a technical system (its function and importance);

2. A model for the deterioration of the system over time and possible consequences for the system;

3. A description of the information available on the system and the actions available to management;

4. An objective function and an optimization technique that helps to find the best balance.

These maintenance optimization models produce different results. First, policies can be evaluated and compared to the characteristics of cost-effectiveness and reliability.
Wang [23] presents an extensive review of maintenance optimization policies. Maintenance optimization studies prior to 2002 mainly considered timebased maintenance configurations. Syan \& Ramsoobag [24] state that modern maintenance optimization decisions are complex problems that need to satisfy multiple and conflicting criteria. With the increase in applications and recent advances in MultiCriteria Optimization (MCO) approaches, a review is needed to group and categorize these advances in the field of maintenance. Jonge \& Scarf [25] says that optimization applied to maintenance comprises the development and analysis of mathematical models that aim to improve or optimize maintenance policies. A study on the substantial developments in the field of maintenance optimization is fully demonstrated in [23].

In order to validate the effectiveness of decision models, Bousdekis et al. [26] prove that an event-driven proactive decision model is possible for joint predictive maintenance and optimization of the spare parts inventory, which addresses the "Detect" "preventdecide-act" model phase that can be incorporated into an EDA (Event Oriented Architecture) for processing time within the framework of the concept of electronic maintenance. Zhou, Qi \& Liu [27] show some drawbacks that the ideal maintenance policy is, in fact, a monotony of control limits, in which the control limits decrease monotonously with the age of the system; but, other studies expose some solutions like Zhao et al. [28], that propose a predictive maintenance policy based on process data, demonstrating that, when compared to traditional preventive maintenance strategies, their strategy have adaptability and effectiveness to the deterioration of the system. Among the techniques presented in [14], there are five non-invasive techniques used for the management of predictive maintenance such as monitoring vibrations, monitoring process parameters, thermography, tribology and visual inspection. Predictive techniques can vary, as mentioned in [29]: lubricant analysis; vibration analysis; thermography; penetrating liquids; radiography; ultrasound; corrosion control; etc.

There is a current concern in making predictive diagnostics to avoid unwanted costs of maintenance and production, having industries and researchers bet on their resources to solve this dilemma. One of the main focuses of multivariate analysis is the search for models that best apply to a forecast or even explain the behaviour of the variables studied. In this section, applications of multivariate models are presented in the scope of engineering, with a focus on asset management during the life cycle monitoring production and maintenance management to reduce unwanted costs. The reduction of expenses in the industrial sphere is 
a great challenge in which it involves a chain of responsibility from the worker who is fully with the machine passing through the managers and reaching the researchers, who have as challenge the way to respond effectively to these events unwanted. Vatn, Hokstad \& Bodsberg [30] present a global approach to maintenance optimization that requires knowledge in several fields; for example, decision theory, risk analysis, reliability and maintenance modelling. The authors also refer that, in order to perform maintenance optimization, it is generally not feasible to carry out a complete risk analysis, and the effect of the chosen maintenance program on safety, that can be assessed by a somewhat simplified approach, considering only a very limited number of scenario representatives. Besnard et al. [31] present an opportunistic maintenance optimization model for offshore wind energy systems. The model takes advantage of wind forecasts and corrective maintenance activities to perform preventive low cost maintenance tasks. Pinto \& Nascif [32] refer that, sometimes, the increase in reliability is done through the expense of availability. This suggests a threefold restriction between quality, availability, and reliability of maintenance, directly influencing costs (Figure 2).

Maintence Quality

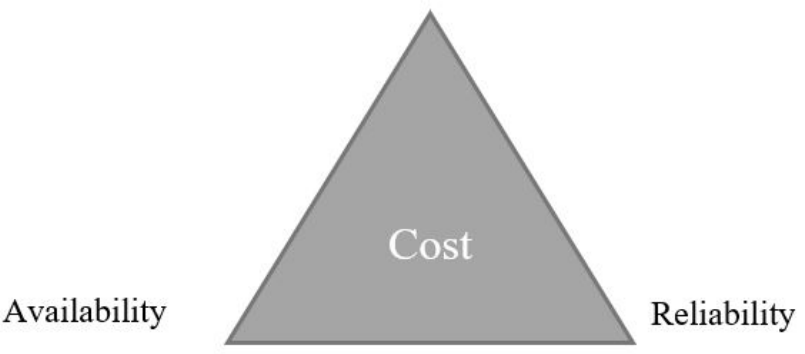

Figure 2: Triple maintenance constraint [32]

Maintenance plays a key role in reliability, availability, product quality, risk reduction, greater equipment efficiency and safety [33]. According to Kershaw \& Robertson [34], predictive maintenance works with periodic monitoring of component conditions, instead of replacing them, which means better data, increasing plant productivity and preventing disastrous failures. For example, Chang et al. [35] explore the optimization of maintenance, presenting a method that incorporates information in real time about production conditions and machine failure. Carnero [36] states that Predictive Maintenance can provide an increase in safety, quality and availability in industrial plants. The graph shown in Figure 3 illustrates that continuous investments in preventive maintenance reduce failure costs and, as a consequence, a decrease in the total maintenance cost, in which preventive maintenance costs are added to the failure costs. However, the graph also shows that, from the ideal point of investment in preventive maintenance, more investments bring few benefits to reduce the cost of failures and end up increasing the total cost, which is what the maintenance policy takes into account.

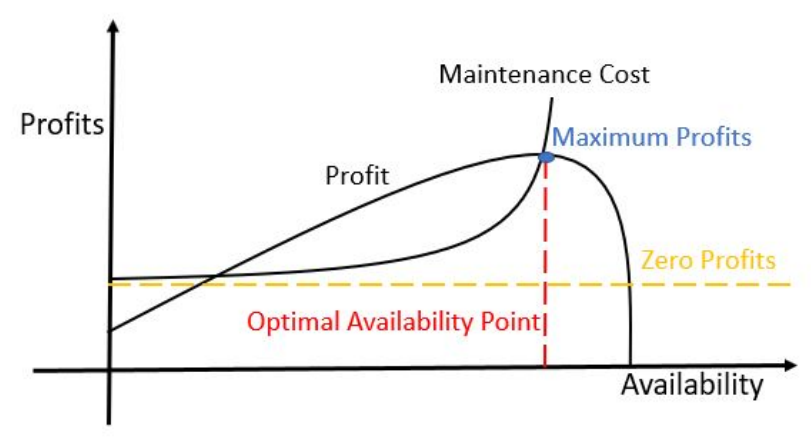

Figure 3: Graph of cost versus maintenance level [37]

However, the creation of a Predictive Maintenance Program is a strategic decision that, until now, lacks an analysis of the problems related to installation, management and control. According to Shin \& Jun [38], when it is a high-value asset, the Operation and Maintenance (O\&M) phase requires heavy charges and more effort than in the installation (construction) phase, as these assets have a useful life that any unexpected event of the asset during that period causes catastrophic damage to the industry.

\section{Some specific approaches}

\subsection{Opportunistic Maintenance}

In order to fulfill the maintenance objectives, the company needs management skills to integrate people, policies, equipment and practices. It also needs adequate engineering and technology [39]. Colledani, Magnanini \& Tolio [40] state that Opportunistic Maintenance (OM) is an effective strategy to reduce interference between maintenance and production operations in multi-stage manufacturing systems and its application in the industry is still limited due to the complexity of predicting its impact on system performance. The maintenance of opportunities is theoretically adjusted automatically; if insufficient opportunities arise, the average delay increases and failures increase until a balance is reached, but there are minimal conditions for a given age renewal schedule and the natural balance may not be economically ideal [41]. Takahashi [42], thorough investigation of these opportunities and their occurrences, comes to next questions:

- When opportunities arise, which machines allow for other simultaneous repairs? 
- What opportunities are needed, when do they arise, and for how long?

For Takahashi [42], Maintenance Management must consider some points to restructure a company and prepare it for future challenges, always with the participation of all employees:

1. Restrict investments in unnecessary equipment;

2. Make the most of existing equipment;

3. Improve the rate of use of equipment for production;

4. Guarantee the quality of the product, through the use of the equipment;

5. Reduce low-cost labour, improving equipment;

6. Reduce the cost of energy and material purchased, through innovations in equipment and improvements in methods of use.

Iung, Levrat \& Thomas [43] show that the numerical results of the study presented in the paper, by properly defining the parameters of opportunistic maintenance actions, it is possible to obtain an effective synchronization of preventive maintenance and production operations, preserving the conditions of the machine and meeting the production goals. The superiority of Condition Based Maintenance remains uncertain in multicomponent systems, in which opportunistic maintenance strategies can be applied [44].

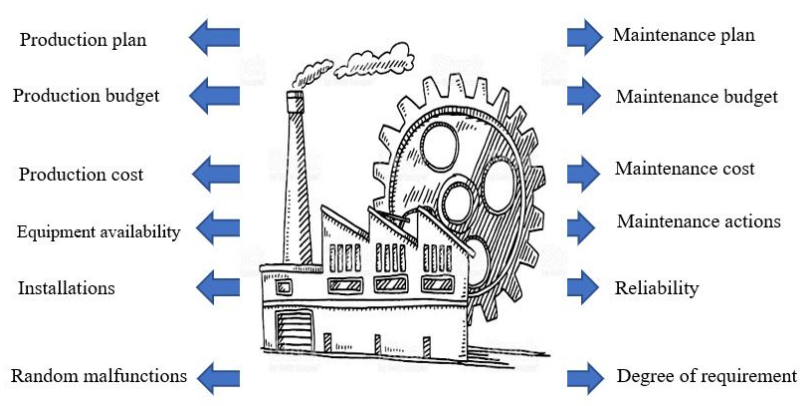

Figure 4: production / maintenance interface [20]

Compared to the static opportunistic maintenance strategy and the strategy without considering opportunistic maintenance, the total cost of maintenance and inventory of the dynamic opportunistic maintenance strategy shows a decline of $10.927 \%$ and $18.304 \%$, respectively [45]. Vu et al. [46] mention that the structure of the system, the maintenance opportunity and its support, as well as the economic dependence, are important issues that must be considered when making maintenance decisions.
In CBM, the opportunistic zone is defined as (part of) the P-F interval (Figure 5) that is part of the degradation curve. It starts at the point where a potential failure can be detected (P) and ends at the moment when the failure occurs (F) [47].

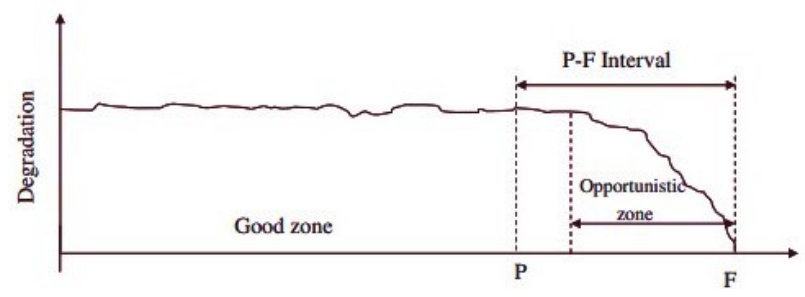

Figure 5: Opportunistic maintenance zone in CBM policy [44]

In case of opportunistic maintenance, the maintenance activities can begin at an arbitrary point within the opportunistic zone (which is equal to or less than the P-F interval). This opportunistic zone is the period during which the degradation started, without leading to a fatal shutdown of the component. Within the opportunistic zone, Planned Maintenance (PM) activities can be carried out against PM costs [44]. Rao [48] demonstrates that policies with various opportunistic maintenance ages for each increasing failure rate component are better with respect to policies with a single opportunistic maintenance age for each component. Performing preventive maintenance, even when there is no opportunity, can have a detrimental effect. It can be said that opportunistic maintenance policies of the type $(n, \infty)$ are sufficient in the case of systems with a large number of components [48]. Zhou, Xi $\&$ Lee [49] studied a dynamic programming method in which decisions are based on a combination of OM cost savings and penalty costs and stated that an ideal maintenance practice is determined by maximizing the savings of cumulative short-term costs. Ding \& Tian [50] proposed a method for making opportunistic maintenance decisions by comparing the age of a given component with a limit defined by some percentage of the Mean Time To Failure (MTTF). Dekker [1] developed a model to determine the ideal age for opportunistic maintenance when the opportunity follows the Homogeneous Process of Poisson (HPP) [51] with the adaptive opportunistic maintenance model, based on the forecast of the conditions of a railroad, demonstrating that the adaptive opportunistic maintenance strategy has a lower cost per unit of time than systematic preventive maintenance. Truong Ba et al. [52], in their results indicate that significant savings can be achieved considering OM. In addition, it is shown that the new consideration of partial opportunities significantly increases the benefit of OM. 
Among preventive maintenance control policies, opportunistic maintenance is an effective strategy for reducing the impact of maintenance operations on multistage manufacturing systems [43]. Under the scope of Operations Management, one of the great challenges is to give them as occurrences of unexpected failures, which is why numerous studies have emerged with the main objective of avoiding these unexpected events such as Multivariate Analysis and Neural Networks, among others.

\subsubsection{Multivariate Analysis}

One of the main focuses of Multivariate Analysis is the search for models that best apply to a forecast or even explain the behaviour of the variables studied. Multivariate data consists of observations about several different variables for various individuals or objects. Such data immerse in all branches of science, such as Psychology, Education, Geology, Social Sciences, Engineering, Ergonomics, etc. The multivariate method has become an increasingly important area of statistics. In fact, the vast majority of the data is multivariate [53]. The multivariate model also aims at data reduction or structural simplification, classifying, and grouping, investigating the dependency among variables, predicting, and formulating hypotheses [54]. Simoglou, Martin \& Morris [55] extended the existing methodologies to monitor dynamic processes; as it is multivariate, they considers the effect of exogenous inputs, providing additional monitoring methods and appropriate control limits when the serial correlation is present in the data system. There are several techniques for analysing multivariate data. Among them, factor analysis, multiple regression and multiple correlations, multiple discriminant analysis, multivariate analysis of variance and covariance, joint analysis, canonical correlation, cluster analysis, and scheduling. Barker \& Newby [56] presented a study focused on the problem of determining the inspection and maintenance strategy of a system whose state is described by a multivariate stochastic process.

Chatfield \& Collins [53] show that the general point that multivariate analyses tend to be concerned with finding relationships, not only among variables but also among individuals. Based on this, the opportunity arises to include computer technology as Internet of Things (IoT).There is also an extremely important tool, the multivariate control charts, which provides powerful methods to detect out of control situations and to diagnose causes; for example, Hotelling [57] presents a multivariate control plot procedure that is based on the most recent observation - it is insensitive to small and moderate changes in the mean vector. The difficulty of interpreting an out-of-control signal on a multivariate control chart was widely dis- cussed by Chua and Montgomery [58], Alt [59], Doganaksoy et al. [60], Lowry et al. [61] and Haq [62]. The procedures of the multivariate control chart are often considered for use in cascade-type processes, such as those found in the process industries [63].

Multivariate models are a family of several models in which each one has its applicability depending on the behaviour of the data to be analysed. Wang [64] presents a design and an optimization of simulationbased multivariate Bayesian control graph for maintenance applications based on conditions. It combines the use of the concept of delay time and Bayesian theory to develop the posterior probability function of the underlying state, given the history of observed monitoring information.

\subsubsection{Neural Networks}

Within the scope of the development of Artificial Intelligence, there was a promising development, with a high degree of success, with regard to the applications of neural networks in the detection of defects in energy systems, as mentioned in [65], [66] and [67]. According to Lippmann [68], Artificial Neural Networks have been widely used for pattern recognition due to their ability to generalize and respond to unexpected patterns. The main strength of Neural Networks is the ability to recognize patterns in incomplete or "noisy" data. Regarding the forecast, this tool has presented satisfactory answers. Cadenas \& Rivera [69] present a study of wind speed forecast in the region of La Venta, Oaxaca, Mexico, using various techniques of Artificial Neural Networks, with data resources collected, making possible to verify the importance of this tool in relation to the precision.

Jolliffe [70] mentions in his work that the most common application in Principal Component Analysis (PCA) is to reduce, with minimal loss of information, the dimensions of the data varieties. Generally, these data sets constitute a large set of correlated variables that are transformed into a new set of variables, called Principal Components. Not everything is $100 \%$ ideal. Crupi, Guglielmino \& Milazzo [71] present one of the disadvantages of the neural network that requires training.

Bansal, Evans \& Jones [72] exposed a predictive maintenance system in real-time for machine systems based on the neural network approach. The use of simulated data, generated by an experimentally validated simulation model, proved to be effective.

In the study of hydraulic pumps presented in [73], there was a preference by the choice of MultiLayer Perceptron (MLP) neural networks, with greater capacity to be suitable for the classification of patterns. The study provided by Ni \& Wang [74] mentions the effectiveness of the prediction of the Multilayer Feed-forward Neural Networks (MFNNs); the Neu- 
ral Network models showed a high precision in their results. Firat \& Gungor [75] go in the same research line reaching the results, indicating that the General regression neural network can be successfully applied to predict the depth of cleaning around the pillars of the circular bridge.

AlGhazzawi \& Lennox [76], in their study, exposed that static PCA techniques were not suitable for the development of a simple process monitoring system that would allow process operators to quickly and easily identify any sources of abnormalities in the process. Zaranezhad, Mahabadi \& Dehghani [77], in a numerical analysis with respect to the result of neural networks, present a comparison of the precision model demonstrating that the perceptron neural networks had a prediction accuracy of $90.9 \%$ with a prediction accuracy rate of $96.19 \%$; the neuralGA model obtained the highest forecast accuracy of $95.9 \%$ and an accuracy of $96.7 \%$. In a forecasting study presented in [78], it is said that its quality of RNA prediction can be improved by expanding the training data sets and optimizing the construction of the network. Rao [79] also states that the results will depend on the collection of high-quality data.

Gilabert \& Arnaiz [80] present a case study based on a Neural Network, where it is possible to find a predictive maintenance solution for non-critical machines. The results indicate the feasibility of partial solutions in monitoring and diagnosis.

Fu et al. [81] present a form of identification and diagnosis based on Artificial Neural Network (ANR) in the electro-hydraulic servomechanisms of a hydroelectric unit. Experimental tests show that the proposed strategy can guarantee optimal performance. According to the study presented in [82], predictive maintenance is already used or will be used by $83 \%$ of production companies; it has been a valuable application of the Internet of Things (IoT) mainly on the factory floor.

According to the CXP Group report, the Digital Industrial Revolution with Predictive Maintenance showed that the level of use of predictive maintenance is being used by $91 \%$ of manufacturers [ 83$]$; it can be verified that the reduction in the repair time of $93 \%$ of the companies pointed to the improvement of the old industrial infrastructure as the main objective of their predictive maintenance initiatives.

Javadpour \& Knapp [84] present an implementation of a predictive neural network for use as an operator assistance fault diagnosis with high forecasting accuracy in an automated manufacturing environment. The network was able to correctly classify three different fault categories with a performance rate greater than $99 \%$ in standards and $100 \%$ in failures. As a result of implementing adequate tools, ans according to a PWC report [85], predictive maintenance in facto- ries could:

- Reduce the cost by $12 \%$;

- Improve uptime by $9 \%$;

- Reduce safety, health, environment and quality risks by $14 \%$;

- Increase the useful life of an old asset by 20

\section{Proposed approaches}

The previous sections show that maintenance policies play an essential role in the operations management. One of the big challenges of Operations Management science is to avoid several losses in the goods' manufacturing process, avoiding unwanted losses; most of these failures are classified as random but, through the reliability function and related tools, it is possible to exploit the maintenance capacity through its policies; for example, the predictive maintenance policy, supported by the math tools referred in the past section, can make predictions with results with a high degree of rigour - but, it must be emphasized that these results depends on the quality of the data available.

With these tools, the proposal is to improve the asset monitoring system using the portfolios provided by the asset management system; in this perspective, it is possible to automate the maintenance system using the concept that emerged in 1999, and whose main objective is to make equipment autonomous and intelligent enough, aiming they do not need human intervention: this concept is supported by the IoT [86]. Applying IoT in the industry, it becomes possible, for maintenance stops, to converge to an optimal reduction in intervention times and, consequently, in costs, which implies making the process more profitable.

The purpose of future developments is to explore the predictive maintenance policy in order to avoid bottleneck phenomena, making the production process more versatile, regarding to capacity readjustment. Neural Networks will be used to support the classification of groups of variables in order to understand them, and the multivariate analysis applied to forecast, with the main focus on Machine Learning (ML). Since maintenance is an important branch in the production process, Big Data analysis, Machine Learning and the IoT, using real-time forecasting in the manufacturing equipment, will make possible to predict failures at run time, aiming to launch Working Orders before the fault happens [87]. For a first test, the technologies of Figure 6 will be applied.

\section{Discussion}

The present article brings an overview of the linked literature Optimizing production by maximizing the availability of assets, when it comes to optimizing 

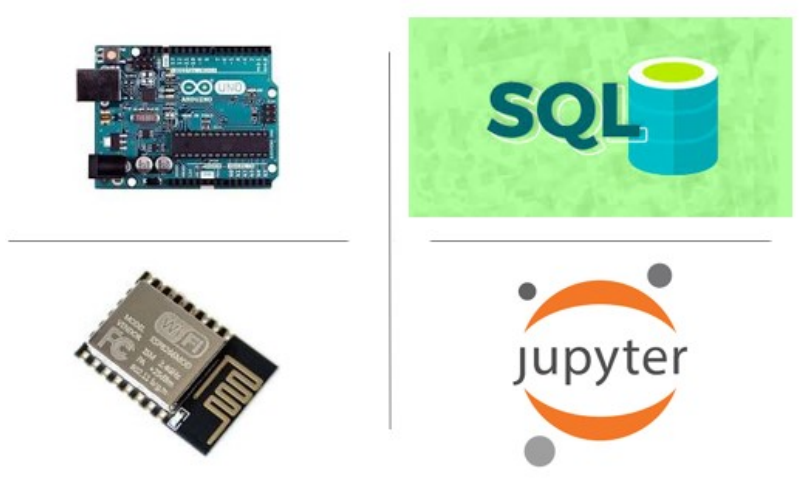

Figure 6: Support technologies.

production it is necessary to have accessibility than that the trailer behind, for this reason our study brings the reader a location of concepts that directly influences the study presented on this topic.

What can be seen in many studies in this area about a conflict between the investment limit and the return that a prediction maintenance system can bring to an entity? Regarding the prediction methods by multivariate models, there are very few, and there is a gap regarding the automation of prediction through this model. Regarding neural networks, there is a wide range of studies, this does not mean that it presents a significant advantage over multivariate analysis, each of which has particular advantages.

When it is possible to have a timely response to make decisions that avoid making bad decisions in any department of a company, the big challenge is to reduce the time to act in the face of events such as failures. Therefore, there are mathematical and computational tools that, together, can bring a great benefit, such as obtaining data through IoT technology and exploring them for possible event prediction. Finally, we will have an optimized process, as these failures can be controlled and resolved within a restricted time window. As the failure of a machine will depend on many variables to be extinguished, such as vibration, temperature and noise, among others; one variable may be related to another or not, which makes multivariate analysis an ideal tool for this study. Currently, some "software" and their respective languages show that many have limitations in terms of their programming. Based on the tools presented in this paper, it is possible to avoid unwanted events; if we work with probabilities, hence the great strength of the predictive maintenance policy, that arises because it reduces the probability of equipment failures; its domain is covering the production system and the values associated with the production is the availability of the equipment that is related with its faults. Since the total cost is the sum of all costs related to production, this means that the reduction in one of them reflecting the reduction of the total cost. According to Yip, Fan \& Chiang [88], the time series models General Regression Neural Network (GRNN) and the Box-Jenkins, usually describe the behaviour and predict the costs associated with maintaining different categories of equipment at an acceptable level of precision. Mateus, Margalho \& Farinha [89] presented in their studies the disadvantages that the ARMA time series model presents in relation to the forecast when faced with oscillatory data (dummy variables).

During this research, it was possible to verify that the multivariate analyses showed promising results in the researches done, solving problems that were previously unthinkable because they had a solution or approaches to solutions of a great result. With this multivariate analysis, these solutions are a good part of the performance and versatility of the "software" developed for these purposes. During the problem-solving process, it is necessary to go through several assumptions. According Renwick [90] Most benefits the predictive maintenance programs include not only evident cost benefits, such as reducing machinery downtime and production losses, but also the more subtle long-term cost benefits which can result from accurate maintenance scheduling.

The importance of Maintenance in a production system has been validated and a concern to respond in a timely manner to unwanted stops on a manufacturing line. To solve problems, knowing the variables in question must be one of the important factors, knowing the right time for intervention, without forget the life cycles of all parts that make up a complex system, with complex problems joining simple problems.

\section{Conclusions}

The paper presents a global approach about the maintenance in production results, and that it is possible to validate the use and efficiency of several tools in solving problems related to the increase maintenance efficiency. In the Maintenance space, some studies were applied to solutions in which it was possible to detect and diagnose the failure based on this approach, and also verifying how efficient the Neural Networks and the Principal Component Analysis are. The accumulated costs associated with factory failure have a high level of significance. For this reason, maintenance approaches have evolved to respond to these dilemmas, such as Predictive Maintenance (PM), which has always shown the ability to evolve to maintain integrity in companies, generating information about the conditions of the equipment; this data allows maintenance to be effective.

The real cost of implementing and maintaining a predictive maintenance program is not the initial cost of the system. Instead, it is the annual labour costs and 
indirect costs associated with the acquisition, storage, trends, and analysis of the data necessary to determine the operational condition of the plant's facilities. In this area, predictive maintenance systems present a greater variation in their capacity, automation in data acquisition and storage, etc.; this will reduce operating costs.

\section{References}

[1] R. Dekker, "Applications of maintenance optimization models: a review and analysis," vol. 51, no. 3, pp. 229-240, 1996-03. [Online]. Available: https://linkinghub.elsevier. com/retrieve/pii/0951832095000763

[2] A. Garg and S. Deshmukh, "Maintenance management: literature review and directions," vol. 12, no. 3, pp. 205-238, 2006-07. [Online]. Available: https://www.emerald.com/insight/content/doi/ 10.1108/13552510610685075/full/html

[3] A. Sharma, G. Yadava, and S. Deshmukh, "A literature review and future perspectives on maintenance optimization," vol. 17, no. 1, pp. 5-25, 2011-01-01, publisher: Emerald Group Publishing Limited. [Online]. Available: https://doi.org/10.1108/13552511111116222

[4] A. Alrabghi and A. Tiwari, "State of the art in simulation-based optimisation for maintenance systems," vol. 82, pp. 167-182, 2015-04. [Online]. Available: https://linkinghub.elsevier. com/retrieve/pii/S0360835214004513

[5] L. Wang, J. Chu, and W. Mao, "An optimum condition-based replacement and spare provisioning policy based on markov chains," vol. 14, no. 4, pp. 387-401, 2008-09-26. [Online]. Available: https://www.emerald.com/insight/content/doi/ 10.1108/13552510810909984/full/html

[6] S. Duffuaa, A. Kolus, U. Al-Turki, and A. ElKhalifa, "An integrated model of production scheduling, maintenance and quality for a single machine," vol. 142, p. 106239, 2020-04. [Online]. Available: https://linkinghub.elsevier. com/retrieve/pii/S0360835219307089

[7] S. Amari and L. McLaughlin, "Optimal design of a condition-based maintenance model," in Annual Symposium Reliability and Maintainability, 2004 - RAMS. IEEE, 2004, pp. 528-533. [Online]. Available: http://ieeexplore.ieee.org/document/1285501/
[8] C. Chu, J.-M. Proth, and P. Wolff, "Predictive maintenance: The one-unit replacement model," vol. 54, no. 3, pp. 285-295, 1998-05. 「Online]. Available: https://linkinghub.elsevier. com/retrieve/pii/S0925527398000048

[9] J. Khalil, S. M. Saad, and N. Gindy, "An integrated cost optimisation maintenance model for industrial equipment," vol. 15, no. 1, pp. 106-118, 2009-03-27. [Online]. Available: https://www.emerald.com/insight/content/doi/ 10.1108/13552510910943912/full/html

[10] A. Gill, "Optimisation of the technical object maintenance system taking account of risk analysis results," vol. 19, no. 3, pp. 420-431, 2017-06-12. [Online]. Available: http://www. ein.org.pl/sites/default/files/2017-03-13.pdf

[11] A. H. C. Tsang, A. K. S. Jardine, J. D. Campbell, and J. V. Picknell, Reliability centred maintenance : a key to maintenance excellence. Dept. of Manufacturing Engineering and Engineering Management, City University of Hong Kong, 2000, accepted: 2016-07-29T04:04:40Z Journal Abbreviation: Reliability-centered maintenance: towards excellence. [Online]. Available: http://ira.lib.polyu.edu.hk/handle/10397/54245

[12] K. K. Boyer and C. McDermott, "Strategic consensus in operations strategy," vol. 17, no. 3, pp. 289-305, 1999-03. [Online]. Available: http://doi.wiley.com/10.1016/ S0272-6963(98)00042-4

[13] B. Dhillon, Maintainability, Maintenance, and Reliability for Engineers, 0th ed. CRC Press, 2006-03-27. [Online]. Available: https://www. taylorfrancis.com/books/9781420006780

[14] R. K. Mobley, An Introduction to Predictive Maintenance(Plant Engineering), 2nd ed. Butterworth-Heinemann, 2002-10-24, googleBooks-ID: SjqXzxpAzSQC.

[15] K. O. Cua, K. E. McKone, and R. G. Schroeder, "Relationships between implementation of TQM, JIT, and TPM and manufacturing performance," vol. 19, no. 6, pp. 675-694, 2001-11. [Online]. Available: http://doi.wiley. com/10.1016/S0272-6963(01)00066-3

[16] J. Wang, Y. Lu, Y. Yang, and T. Mao, "Thermodynamic performance analysis and optimization of a solar-assisted combined cooling, heating and power system," vol. 115, pp. 49-59, 2016-11-15. [Online]. Available: http://www.sciencedirect.com/science/ article/pii/S0360544216312154 
[17] L. G. Hernández-Pérez, C. Ramírez-Márquez, J. G. Segovia-Hernández, and J. M. PonceOrtega, "Simultaneous structural and operating optimization of process flowsheets combining process simulators and metaheuristic techniques: The case of solar-grade silicon process," p. 106946, 2020-05. [Online]. Available: https://linkinghub.elsevier.com/retrieve/ pii/S0098135419312402

[18] H. R. Rao and B. P. Lingaraj, "Expert systems in production and operations management: Classification and prospects," vol. 18, no. 6, pp. 80-91, 1988-12. [Online]. Available: http://pubsonline.informs.org/doi/abs/10. 1287/inte.18.6.80

[19] M. Dorigo, G. D. Caro, and L. M. Gambardella, "Ant algorithms for discrete optimization," vol. 5, no. 2, pp. 137-172, 1999-04. 「Online]. Available: http://www.mitpressjournals. org/doi/10.1162/106454699568728

[20] C. Blum and A. Roli, "Metaheuristics in combinatorial optimization: Overview and conceptual comparison," vol. 35, no. 3, pp. 268-308, 2003-09-01. [Online]. Available: http://portal. acm.org/citation.cfm?doid=937503.937505

[21] S. Alaswad and Y. Xiang, "A review on condition-based maintenance optimization models for stochastically deteriorating system," vol. 157, pp. 54-63, 2017-01. [Online]. Available: https://linkinghub.elsevier.com/retrieve/ pii/S0951832016303714

[22] J. Nocedal and S. Wright, Numerical Optimization, 2nd ed. Springer Science \& Business Media, 2006-06-06.

[23] H. Wang, "A survey of maintenance policies of deteriorating systems," vol. 139, no. 3, pp. 469-489, 2002-06. [Online]. Available: https://linkinghub.elsevier.com/retrieve/ pii/S0377221701001977

[24] C. S. Syan and G. Ramsoobag, "Maintenance applications of multi-criteria optimization: A review," vol. 190, p. 106520, 2019-10. [Online]. Available: https://linkinghub.elsevier. com/retrieve/pii/S0951832018313759

[25] B. de Jonge and P. A. Scarf, "A review on maintenance optimization," $p$. S0377221719308045, 2019-09. [Online]. Available: https://linkinghub.elsevier.com/retrieve/ pii/S0377221719308045
[26] A. Bousdekis, N. Papageorgiou, B. Magoutas, D. Apostolou, and G. Mentzas, "A proactive event-driven decision model for joint equipment predictive maintenance and spare parts inventory optimization," vol. 59, pp. 184-189, 2017. [Online]. Available: https://linkinghub.elsevier. com/retrieve/pii/S2212827116309490

[27] B. Zhou, Y. Qi, and Y. Liu, "Proactive preventive maintenance policy for buffered serial production systems based on energy saving opportunistic windows," vol. 253, p. 119791, 2020-04-20. [Online]. Available: http://www.sciencedirect.com/science/ article/pii/S095965261934661X

[28] Z. Zhao, F.-1. Wang, M.-x. Jia, and S. Wang, "Predictive maintenance policy based on process data," vol. 103, no. 2, pp. 137-143, 2010-10-15. [Online]. Available: http://www.sciencedirect.com/science/ article/pii/S016974391000119X

[29] D. J. Edwards, G. D. Holt, and F. Harris, "Predictive maintenance techniques and their relevance to construction plant," vol. 4, no. 1, pp. 25-37, 1998-03. [Online]. Available: https://www.emerald.com/insight/content/doi/ 10.1108/13552519810369057/full/html

[30] J. Vatn, P. Hokstad, and L. Bodsberg, "An overall model for maintenance optimization," vol. 51, no. 3, pp. 241-257, 1996-03. [Online]. Available: https://linkinghub.elsevier. com/retrieve/pii/0951832095000550

[31] F. Besnard, M. Patrikssont, A.-B. Strombergt, A. Wojciechowskit, and L. Bertling, "An optimization framework for opportunistic maintenance of offshore wind power system," in 2009 IEEE Bucharest PowerTech, 2009-06, pp. 1-7.

[32] A. K. Pinto and J. Nascif, Maintenance Strategic Function, 2nd ed. Qualitymark, 2001.

[33] M. Zaeri, J. Shahrabi, M. Pariazar, and A. Morabbi, "A combined multivariate technique and multi criteria decision making to maintenance strategy selection," in 2007 IEEE International Conference on Industrial Engineering and Engineering Management. IEEE, 2007-12, pp. 621-625. [Online]. Available: http://ieeexplore.ieee.org/document/4419264/

[34] J. Kershaw and B. Robertson, "Condition-based maintenance program increases production, reduces costs," pp. 34-36, 1985. 
[35] Q. Chang, J. Ni, P. Bandyopadhyay, S. Biller, and G. Xiao, "Maintenance opportunity planning system," vol. 129 , no. 3, pp. 661-668, 2007-06-01, publisher: American Society of Mechanical Engineers Digital Collection. [Online]. Available: https://asmedigitalcollection. asme.org/manufacturingscience/ article/129/3/661/462149/ Maintenance-Opportunity-Planning-System

[36] M. Carnero, "Selection of diagnostic techniques and instrumentation in a predictive maintenance program. a case study," vol. 38, no. 4, pp. 539-555, 2005-01. [Online]. Available: https://linkinghub.elsevier.com/retrieve/ pii/S0167923603001283

[37] A. Murty and V. Naikan, "Availability and maintenance cost optimization of a production plant," vol. 12, no. 2, pp. 28-35, 1995-03. [Online]. Available: https://www.emerald.com/insight/content/doi/ 10.1108/02656719510080596/full/html

[38] J.-H. Shin and H.-B. Jun, "On condition based maintenance policy," vol. 2, no. 2, pp. 119-127, 2015-04. [Online]. Available: https://linkinghub.elsevier.com/retrieve/ pii/S2288430014000141

[39] L. Pintelon and L. Gelders, "Maintenance management decision making," vol. 58, no. 3, pp. 301-317, 1992-05. [Online]. Available: https://linkinghub.elsevier.com/retrieve/ pii/037722179290062E

[40] M. Colledani, M. C. Magnanini, and T. Tolio, "Impact of opportunistic maintenance on manufacturing system performance," vol. 67, no. 1, pp. 499-502, 2018. [Online]. Available: https://linkinghub.elsevier.com/retrieve/ pii/S0007850618301021

[41] D. J. Sherwin, "Age-based opportunity maintenance," vol. 5, no. 3, pp. 221-235, 1999-01-01, publisher: MCB UP Ltd. [Online]. Available: https://doi.org/10.1108/13552519910282674

[42] Y. Takahashi, TPM/MPT: Total Productive Maintenance, 5th ed. IMAM, 2010.

[43] B. Iung, E. Levrat, and E. Thomas, “"odds algorithm'-based opportunistic maintenance task execution for preserving product conditions," vol. 56, no. 1, pp. 13-16, 2007. [Online]. Available: https://linkinghub.elsevier. com/retrieve/pii/S0007850607000054
[44] J. Koochaki, J. A. Bokhorst, H. Wortmann, and W. Klingenberg, "Condition based maintenance in the context of opportunistic maintenance," vol. 50, no. 23, pp. 6918-6929, 2012-12. [Online]. Available: http://www.tandfonline. com/doi/abs/10.1080/00207543.2011.636924

[45] C. Zhang, W. Gao, T. Yang, and S. Guo, "Opportunistic maintenance strategy for wind turbines considering weather conditions and spare parts inventory management," vol. 133, pp. 703-711, 2019-04. [Online]. Available: https://linkinghub.elsevier.com/retrieve/ pii/S0960148118312606

[46] H. C. Vu, P. Do, M. Fouladirad, and A. Grall, "Dynamic opportunistic maintenance planning for multi-component redundant systems with various types of opportunities," vol. 198, p. 106854, 2020-06-01. [Online]. Available: http://www.sciencedirect. com/science/article/pii/S0951832019309421

[47] J. Moubray, Reliability-Centered Maintenance, 2nd ed. Butterworth-Heinemann, 1997.

[48] B. Rao, "Opportunistic maintenance of multiequipment system: a case study," p. 14, 2000.

[49] X. Zhou, L. Xi, and J. Lee, "Opportunistic preventive maintenance scheduling for a multi-unit series system based on dynamic programming," vol. 118, no. 2, pp. 361-366, 2009-04-01. [Online]. Available: http://www.sciencedirect. com/science/article/pii/S0925527308003472

[50] F. Ding and Z. Tian, "Opportunistic maintenance for wind farms considering multi-level imperfect maintenance thresholds," vol. 45, pp. 175-182, 2012-09-01. [Online]. Available: http://www.sciencedirect.com/science/ article/pii/S0960148112001802

[51] C. Letot, I. Soleimanmeigouni, A. Ahmadi, and P. Dehombreux, "An adaptive opportunistic maintenance model based on railway track condition prediction," vol. 49, no. 28, pp. 120-125, 2016-01-01. [Online]. Available: http://www.sciencedirect.com/science/ article/pii/S2405896316324454

[52] H. Truong Ba, M. E. Cholette, P. Borghesani, Y. Zhou, and L. Ma, "Opportunistic maintenance considering non-homogenous opportunity arrivals and stochastic opportunity durations," vol. 160, pp. 151-161, 2017-04-01. [Online]. Available: http://www.sciencedirect. com/science/article/pii/S095183201631002X 
[53] C. Chatfield and A. J. Collins, Introduction to Multivariate Analysis, a c r c press company ed. Northwestern University, 2000.

[54] R. A. Johnson and D. W. Wichern, Applied multivariate statistical analysis, 6th ed. Pearson Prentice Hall, 2007, OCLC: ocm70867129.

[55] A. Simoglou, E. Martin, and A. Morris, "Statistical performance monitoring of dynamic multivariate processes using state space modelling," vol. 26, no. 6, pp. 909-920, 2002-06. [Online]. Available: https://linkinghub.elsevier. com/retrieve/pii/S0098135402000121

[56] C. Barker and M. Newby, "Optimal nonperiodic inspection for a multivariate degradation model," vol. 94, no. 1, pp. 33-43, 2009-01. [Online]. Available: https://linkinghub.elsevier. com/retrieve/pii/S0951832007001123

[57] H. Hotelling, "Multivariate quality control. techniques of statistical analysis," 1947.

[58] M.-K. Chua and D. C. Montgomery, "Investigation and characterization of a control scheme for multivariate quality control," vol. 8, no. 1, pp. 37-44, 1992, eprint: https://onlinelibrary.wiley.com/doi/pdf/10.1002/q [Online]. Available: https://onlinelibrary.wiley. com/doi/abs/10.1002/qre.4680080107

[59] F. B. Alt, "Multivariate quality control," vol. 6, pp. 110-122, 1985, publisher: John Wiley \& Sons, Inc.

[60] N. Doganaksoy, F. W. Faltin, and W. T. Tucker, "Identification of out of control quality characteristics in a multivariate manufacturing environment," vol. 20, no. 9, pp. 2775-2790, 1991-0101, publisher: Taylor \& Francis eprint: https://doi.org/10.1080/03610929108830667.

[Online]. Available: https://doi.org/10.1080/ 03610929108830667

[61] C. A. Lowry, W. H. Woodall, C. W. Champ, and S. E. Rigdon, "A multivariate exponentially weighted moving average control chart," vol. 34, no. 1, pp. 46-53, 1992-0201, publisher: Taylor \& Francis. [Online]. Available: https://amstat.tandfonline.com/doi/ abs/10.1080/00401706.1992.10485232

[62] A. Haq and M. B. C. Khoo, "Memorytype multivariate control charts with auxiliary information for process mean," vol. 35, no. 1, pp. 192-203, 2019, eprint: https://onlinelibrary.wiley.com/doi/pdf/10.1002/qre.2391.
[Online]. Available: https://onlinelibrary.wiley. com/doi/abs/10.1002/qre.2391

[63] C. A. Lowry and D. C. Montgomery, "A review of multivariate control charts," vol. 27, no. 6, pp. 800-810, 1995-12. [Online]. Available: http://www.tandfonline.com/doi/abs/10. 1080/07408179508936797

[64] W. Wang, "A simulation-based multivariate bayesian control chart for real time conditionbased maintenance of complex systems," vol. 218, no. 3, pp. 726-734, 2012-05. [Online]. Available: https://linkinghub.elsevier. com/retrieve/pii/S0377221711010873

[65] T. Sidhu, H. Singh, and M. Sachdev, "Design, implementation and testing of an artificial neural network based fault direction discriminator for protecting transmission lines," vol. 10, no. 2 , pp. 697-706, 1995-04. [Online]. Available: http://ieeexplore.ieee.org/document/400862/

[66] S. A. Kalogirou, "Applications of artificial neural-networks for energy systems," vol. 67, no. 1, pp. 17-35, 2000.

$6667088_{10}$ A as and S. Kunsman, "A novel approach for ground fault detection," in 57th Annual Conference for Protective Relay Engineers, 2004. IEEE, 2004, pp. 97-109. [Online]. Available: http://ieeexplore.ieee.org/document/ 1287088/

[68] R. P. M. Lippmann, "An introduction to computing with neural nets," vol. 4, pp. 4-22, 1987.

[69] E. Cadenas and W. Rivera, "Wind speed forecasting in the south coast of oaxaca, m $\square$ xico," vol. 32, no. 12, pp. 2116-2128, 2007-10. [Online]. Available: https://linkinghub.elsevier. com/retrieve/pii/S0960148106002801

[70] I. T. Jolliffe, "Principal component analysis," pp. 150-166, 2002.

[71] V. Crupi, E. Guglielmino, and G. Milazzo, "Neural-network-based system for novel fault detection in rotating machinery," vol. 10, no. 8, pp. 1137-1150, 2004-08. [Online]. Available: http://journals.sagepub.com/doi/10.1177/ 1077546304043543

[72] D. Bansal, D. J. Evans, and B. Jones, "A realtime predictive maintenance system for machine systems," vol. 44, no. 7, pp. 759-766, 2004-06. [Online]. Available: https://linkinghub.elsevier. com/retrieve/pii/S0890695504000392 
[73] L. Wang and A. D. Hope, "Fault diagnosis: Bearing fault diagnosis using multi-layer neural networks," vol. 46, no. 8, pp. 451-455, 2004-08. [Online]. Available: http://www.ingentaconnect.com/content/bindt/ insight/2004/00000046/00000008/art00003

[74] H.-G. Ni and J.-Z. Wang, "Prediction of compressive strength of concrete by neural networks," vol. 30, no. 8, pp. 1245-1250, 200008. [Online]. Available: https://linkinghub. elsevier.com/retrieve/pii/S0008884600003458

[75] M. Firat and M. Gungor, "Generalized regression neural networks and feed forward neural networks for prediction of scour depth around bridge piers," vol. 40, no. 8, pp. 731-737, 200908. [Online]. Available: https://linkinghub. elsevier.com/retrieve/pii/S0965997808002056

[76] A. AlGhazzawi and B. Lennox, "Monitoring a complex refining process using multivariate statistics," vol. 16, no. 3, pp. 294-307, 2008-03. [Online]. Available: https://linkinghub.elsevier. com/retrieve/pii/S0967066107001049

[77] A. Zaranezhad, H. Asilian Mahabadi, and M. R. Dehghani, "Development of prediction models for repair and maintenancerelated accidents at oil refineries using artificial neural network, fuzzy system, genetic algorithm, and ant colony optimization algorithm," vol. 131, pp. 331-348, 2019-11-01. [Online]. Available: http://www.sciencedirect. com/science/article/pii/S0957582019310109

[78] J.-T. Zhang and S. Xiao, "A note on the modified two-way MANOVA tests," vol. 82, no. 3, pp. 519-527, 2012-03. [Online]. Available: https://linkinghub.elsevier.com/retrieve/ pii/S0167715211003877

[79] B. K. N. Rao, Handbook of Condition Monitoring, 1st ed. Elsevier Advanced Technology, 1996.

[80] E. Gilabert and A. Arnaiz, "Intelligent automation systems for predictive maintenance: A case study," vol. 22 , no. 5, pp. 543-549, 2006-10. [Online]. Available: https://linkinghub.elsevier. com/retrieve/pii/S0736584506000676

[81] C. Fu, L. Ye, Y. Liu, R. Yu, B. Iung, Y. Cheng, and Y. Zeng, "Predictive maintenance in intelligent-control-maintenancemanagement system for hydroelectric generating unit," vol. 19 , no. 1, pp. 179186, 2004-03. [Online]. Available: http: //ieeexplore.ieee.org/document/1268135/
[82] (2019-05-18) Predictive maintenance: What is the economic value? [Online]. Available: https://www.anodot.com/blog/ predictive-maintenance/

[83] D. M. Milojevic, "Digital industrial revolution with predictive maintenance," p. 32, 2018.

[84] R. Javadpour and G. M. Knapp, "A fuzzy neural network approach to machine condition monitoring," vol. 45, no. 2, pp. 323-330, 200308. [Online]. Available: https://linkinghub. elsevier.com/retrieve/pii/S0360835203000391

[85] "Predictive maintenance - beyond the hype: PdM 4.0 delivers results," p. 19, 2018.

[86] L. M. S. Gouveia, "Impacto da internet of things no lean manufacturing," 2015-06-23. [Online]. Available: https://ubibliorum.ubi.pt/ handle/10400.6/5868

[87] J. Chen, O. Gusikhin, W. Finkenstaedt, and Y.-N. Liu, "Maintenance, repair, and operations parts inventory management in the era of industry 4.0," vol. 52, no. 13, pp. 171-176, 2019. [Online]. Available: https://linkinghub. elsevier.com/retrieve/pii/S2405896319311218

[88] H.-1. Yip, H. Fan, and Y.-h. Chiang, "Predicting the maintenance cost of construction equipment: Comparison between general regression neural network and box-jenkins time series models," vol. 38, pp. 30-38, 2014-03. [Online]. Available: https://linkinghub.elsevier. com/retrieve/pii/S0926580513001921

[89] B. Mateus, L. Margalho, and J. T. Farinha, "Forecasting steel production in germany - an approach through time series, with extrapolation for industrial maintenance," p. 11, 2019.

[90] J. T. Renwick and P. E. Babson, "Vibration analysis - a proven technique as a predictive maintenance tool," vol. IA-21, no. 2, pp. 324-332, 1985-03. [Online]. Available: http: //ieeexplore.ieee.org/document/4157989/

Acknowledgments: The research leading to these results has received funding from the European Union's Horizon 2020 research and innovation programme under the Marie Sklodowvska-Curie grant agreement 871284 project SSHARE, and by the European Regional Development Fund (ERDF) through the Operational Programme for Competitiveness and Internationalization (COMPETE 2020), under Project POCI01-0145-FEDER-029494.

\section{Creative Commons Attribution License 4.0 (Attribution 4.0 International, CC BY 4.0)}

This article is published under the terms of the Creative Commons Attribution License 4.0

https://creativecommons.org/licenses/by/4.0/deed.en_US 